\title{
THE HIPPARCOS OBSERVING PROGRAMME. PERFORMANCES OF THE INPUT CATALOGUE
}

\author{
C. TURON, F. ARENOU, F. CRIFO, A. GÓMEZ AND D. MORIN \\ Observatoire de Paris-Meudon \\ Section d'Astrophysique \\ 92195 MEUDON CEDEX \\ France
}

\begin{abstract}
The Hipparcos observing programme is composed of 118000 selected stars, one QSO (3C273), 48 minor planets, and 2 satellites of major planets. These objects were selected on the ground of scientific proposals submitted by the world-wide astronomical community, while taking into account the specific operating mode of the satellite and using at best its observing possibilities. The content of the stellar part of this observing programme and the way in which the star selection was performed is described in details in a paper accepted for publication in Astronomy \& Astrophysics.
\end{abstract}

The preliminary positions and magnitudes derived from the first observations obtained from Hipparcos allow to check the accuracies claimed by the various sources of groundbased data for single and double stars, and confirm the properties of the Hipparcos Input Catalogue (mean errors on equatorial coordinates of the order of 0.3 arcsec, mean errors on magnitudes varying from 0.04 to 0.3 mag. as a function of magnitude). Comparison with the data received from the Hipparcos star mappers performed by the NDAC Consortium is being published in Astronomy \& Astrophysics.

Less than $0.1 \%$ of the 118000 stars are not successfully observed by the satellite. 